\title{
The porous size distribution obtained and analyzed by free access software
}

\author{
Jocenei Antônio Teodoro de Oliveira ${ }^{1}$, Fabio Augusto Meira Cássaro ${ }^{*} @$, Luiz Fernando Pires ${ }^{1 @}$ \\ ${ }^{1}$ Universidade Estadual de Ponta Grossa, Departamento de Física, Ponta Grossa, PR, Brasil.
}

Received on May 12, 2020. Revised on June 29, 2020. Accepted on July 05, 2020.

\begin{abstract}
This paper presents the description of methods for using in the analysis of the pore size distribution of complex porous media as, for instance, soils. The sequence of analyses presented here can be implemented in classes of post-graduation in physics applied to earth, soil, and environmental sciences. The objective was to present the use, by students and/or researchers, of free access software to analyze images for obtaining some morphological aspects of porous systems. Two methods of obtaining the pore size distribution are presented: the first one is based on ImageJ internal routines, and the second in the study presented in Yang [1]. The results obtained permitted a detailed analysis of three-dimensional(3D) images of porous systems and also obtaining the pore size distribution of the investigated medium. The procedures here introduced can easily be extended for educational purposes in courses related to the investigation of systems with complex internal structures.
\end{abstract}

Keywords: X-ray computed tomography, ImageJ, Soil, Maximum ball, Computed simulation.

\section{Introduction}

Digital image processing for the analysis of complex porous systems, such as rocks and soils, has become popular in different fields of knowledge such as physics applied to earth and environmental sciences $[2,3,4]$. Information about the soil matrix and its pore space is fundamental for studies related to transport of matter and energy through it.

Recent advances in x-ray computed microtomography (CT) have allowed the reconstruction of three-dimensional (3D) inner images of diverse classes of porous materials, with spatial resolutions that extent from micrometers to nanometers $[1,5]$. Using these $3 \mathrm{D}$ detailed images, many different physical processes that occur inside the porous material can be understood and reproduced $[6,7]$.

The number of publications on the use of x-ray CT for the characterization of porous media has significantly increased in the last years. Nowadays, this technique plays an important role in the analysis of the porous system of rocks and soils. The analysis using reconstructed 3D bulk images allows a reliable study of the pore space morphology of soils and rocks $[8,9]$. The continuity of the pores and their distributions in terms of size and shape are examples of properties quantified by non-destructive imaging techniques $[10,11]$.

With the advent of scientific data repositories, it has become relatively easy to access images of x-ray microtomography. These images can be utilized for teaching activities mainly in post-graduation courses of physics applied to earth, environmental, and soil sciences.

*Correspondence email address: fcassaro@uepg.br
In this paper is presented a didactic sequence, to be implemented in post-graduation classes of applied physics and other areas, for the analysis of complex porous systems. To circumvent the need for great investments, it is shown that it can be easily implemented using public domain software.

\section{Material and Methods}

\subsection{Porous medium}

The porous medium here investigated are samples collected from a soil profile in an experimental farm from the Agronomic Institute of Paraná (IAPAR) at Ponta Grossa, Brazil. They have a clayey texture characterized by $578 \mathrm{~g} \mathrm{~kg}^{-1}$ clay, $280 \mathrm{~g} \mathrm{~kg}^{-1}$ silt, and $142 \mathrm{~g} \mathrm{~kg}^{-1}$ sand.

\subsection{The Images and their binarization}

The images here investigate were obtained using a tomographic system model v|tomex|m (GE Measurement \& Control Solutions, Wunstorf, Germany), operating at 180 $\mathrm{kV}$, an operational electric current of $160 \mu \mathrm{A}$ and image time acquisition of $250 \mathrm{~ms}$. The attained resolution of the CT images was $35 \mu \mathrm{m}$ (voxel dimension $35^{3} \mu \mathrm{m}^{3}$ ).

Otsu non-parameterized method (ImageJ 1.42) was utilized for threshold and segment the bi-dimensional (2D) cross-section images. After the thresholding process, they were binarized: pores are white and soil particles are black. 


\subsection{Software for investigating the pore system}

The software used for investigating the Pore Size Distribution (PSD) was ImageJ (1.42) and Yang [1]. Both are based on the maximum ball insertion inside the pore space. The procedure (in a bidimensional image) is schematically presented in Figure 1.

A set of balls with different diameters are used to fulfill the pores of the medium. In Figure 1 only two balls are presented. In both programs, Yang and ImageJ, the pixels associated with each ball are different colored and assigned. In the example of Figure 1, orange balls are associated with larger diameters as blue and magenta are associated with smaller ones. It is worth mentioning that the process used by the software is based on the mercury intrusion procedure, largely employed by students from courses of physics, engineer, and geology for producing the pore size distribution of any porous materials. In this procedure, the mercury is inserted by pressure, little by little (from orange to blue), from the largest to the smallest portion of the pore, until it is filled up.

\subsection{The pore size distribution (PSD)}

After the use of the software, one has to separate the voxels belonging to each founded diameter (color - ball). The summation of all voxels associated with balls of a certain diameter $\left(d^{\prime}\right)$ divided by the volume of the 3D reconstructed volume represents the ratio of pores of this particular diameter $\left(r\left(d^{\prime}\right)\right)$ in the investigated sample (equation 1):

$$
r\left(d^{\prime}\right)(\%)=\frac{100 \sum \operatorname{Voxels}\left(d^{\prime}\right)}{\text { reconstructed volume }}
$$

where: $r\left(d^{\prime}\right)$ is the ratio of pores of a particular diameter $d^{\prime} ; \sum \operatorname{Voxels}\left(d^{\prime}\right)$ is the number of voxels associated with $d^{\prime}$; and reconstructed volume is the total volume of the 3D image (in this study, 860 by 860 by 500 voxels; width, height, and depth, respectively).

\section{Results and Discussion}

In Figure 2 are presented 3D images of the maximum ball procedure applied to one of the investigated samples (named as R1).

It can be seen that the sample under investigation is primarily constituted of small disconnected pores (clusters of small pores). Some of the larger ones are rounded and probably, in the case of soil samples, were produced by dead crop roots or even due to biological (earthworms) activities produced previously of the sample collection. So, with the approach, one can visualize important pore geometrical aspects related to the investigated medium.

Its quantification, using equation 1 , makes it possible to find the pore size distribution related to the investigated sample. Using both approaches, the PSD of the soil sample under investigation is presented in Figure 3.

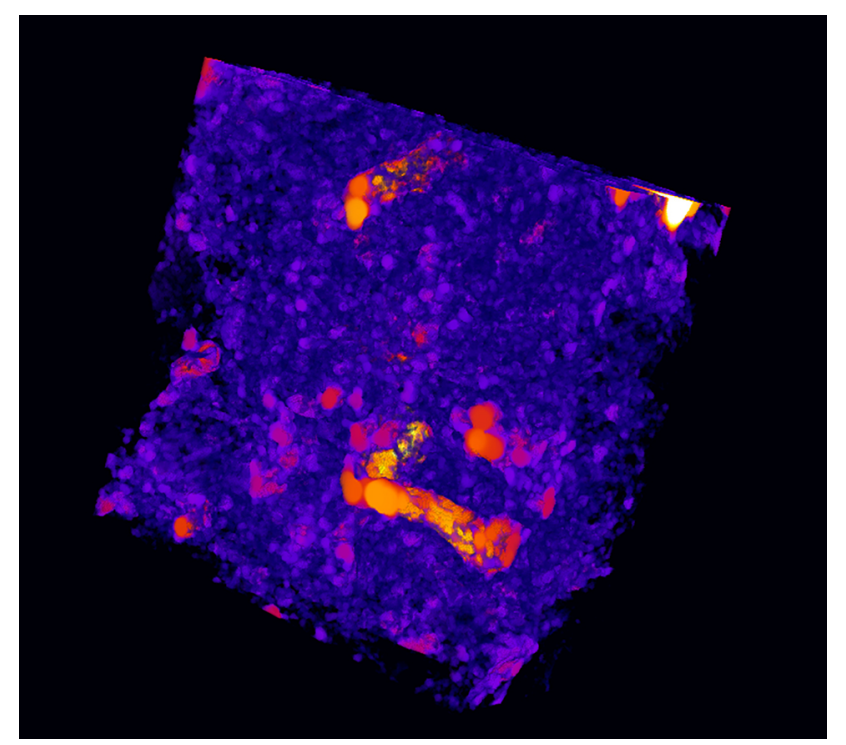

Figure 2: The maximum ball procedure applied to the investigated sample (ImageJ - 1.42). Orange to blue indicates the larger to the smaller investigated pores.

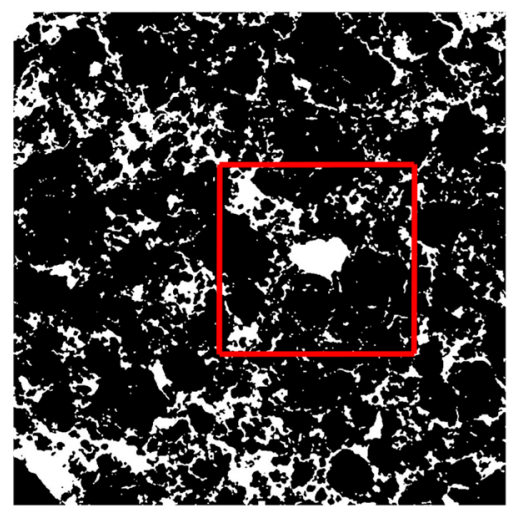

Binarized porous medium

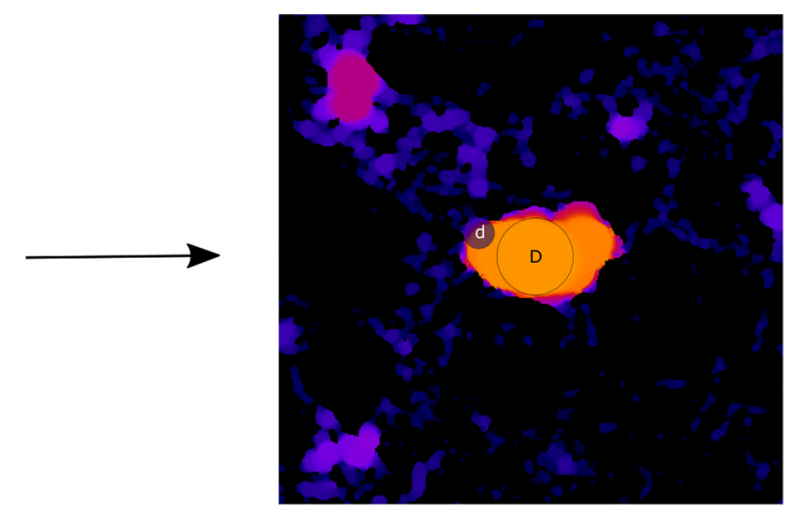

Maximum ball procedure

Figure 1: Left: binarized porous medium. Right: maximum ball insertion procedure showing the largest (D) and the smallest (d) balls associated with a portion (red square) of the investigated image. 
Table 1: Pore size distribution according to Brewer's classification.

\begin{tabular}{lccccc}
\hline ImageJ & Very fine (\%) & Fine (\%) & Medium (\%) & Coarse (\%) & Total (\%) \\
\hline R1 & 26.1 & 0.91 & 0.08 & 0.00 & 27.1 \\
R2 & 14.1 & 0.29 & 0.02 & 0.00 & 14.4 \\
R3 & 13.6 & 0.42 & 0.03 & 0.00 & 14.1 \\
Avg & $17.9(7.1)$ & $0.54(0.33)$ & $0.04(0.03)$ & - & $18.5(7.4)$ \\
\hline Yang & Very fine (\%) & Fine (\%) & Medium (\%) & Coarse (\%) & Total (\%) \\
\hline R1 & 25.8 & 1.82 & 0.12 & 0.00 & 27.7 \\
R2 & 15.5 & 1.68 & 0.11 & 0.00 & 17.3 \\
R3 & 21.5 & 0.39 & 0.00 & 0.00 & 21.9 \\
Avg & $20.9(5.2)$ & $1.30(0.79)$ & $0.08(0.07)$ & - & $22.3(5.2)$ \\
\hline
\end{tabular}

$\mathrm{R}$ stands for replicate. Values between parenthesis represent the standard deviation of the average (Avg)
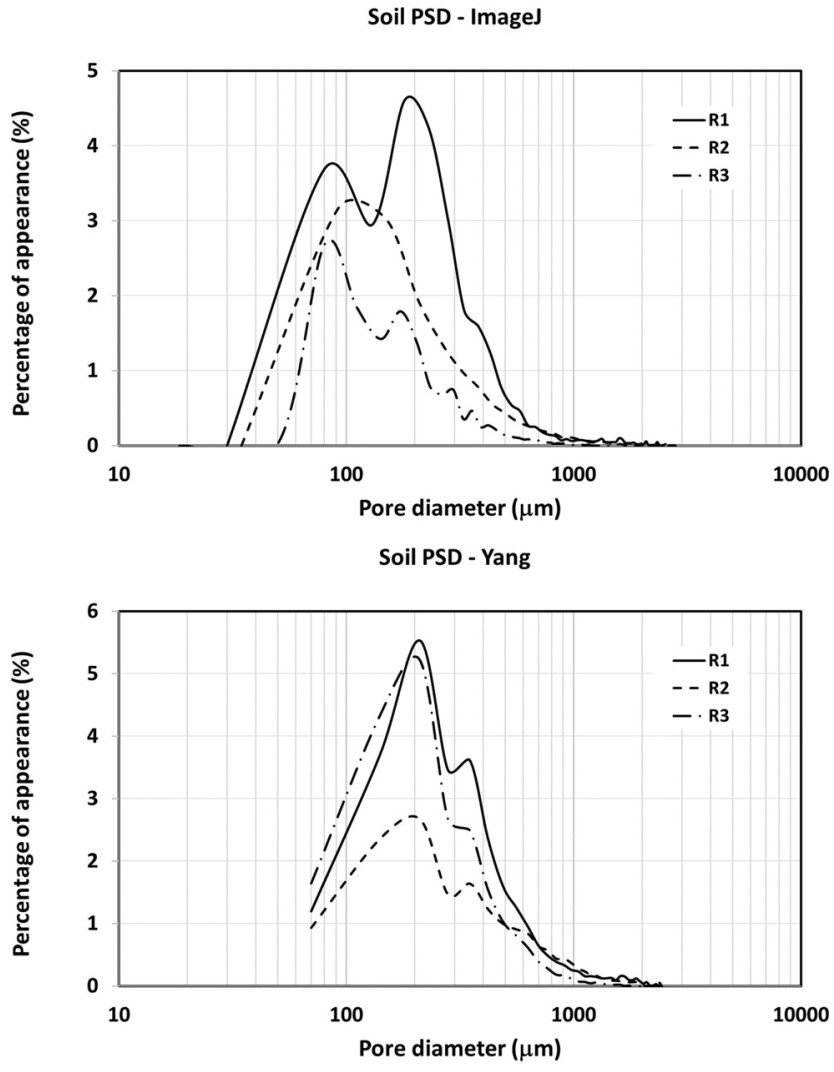

Figure 3: Above: The soil PSD using ImageJ maximum ball procedure. Below: The soil PSD using the Yang maximum ball procedure. R1, R2, and R3 are different samples of the same soil.

It can be seen that the pores of the soil are mainly distributed in the range of diameters between 100 and $1000 \mu \mathrm{m}$. Pores exist between and within small soil portions called soil structural aggregates. Macropores are large soil pores, usually between these aggregates and, micropores usually found within structural aggregates.

According to Brewer [12], soil macropores are divided into four categories according to their characteristic diameter: coarse $(>5000 \mu \mathrm{m})$, medium $(2000-5000 \mu \mathrm{m})$, fine $(1000-2000 \mu m)$, and very fine $(75-1000 \mu m)$. Then, following this classification, the investigated pores are mainly fine and very fine macropores.
From the PSDs, it is also possible to calculate the accessed porosity of the $3 \mathrm{D}$ images according to the range of the investigated pores. It is achieved by summing $\left(r\left(d^{\prime}\right)\right)$ between diameters of interest, for instance, $\mathrm{d} 1$ and $\mathrm{d} 2$, according to equation 2 .

$$
\%(\mathrm{~d} 1, \mathrm{~d} 2)=\sum_{d 1}^{d 2} r\left(d^{\prime}\right)
$$

where: $\%(\mathrm{~d} 1, \mathrm{~d} 2)$ is the percentage of pores between the pre-selected diameters $\mathrm{d} 1$ and $\mathrm{d} 2$; and $\sum_{d 1}^{d 2} r\left(d^{\prime}\right)$ is the summation of ratios of pores between these diameters.

From equation 2 and the classification presented in Brewer [12], the distribution of the examined soil macropores is presented in Table 1.

From both approaches, it is seen that the soil macropores are largely categorized as very fine macropores (characteristic diameters, $75-1000 \mu \mathrm{m}$ ). Also, the maximum ball approaches make it possible to calculate the total accessed porosity of the samples. It is presented, from each approach and soil sample replicate, in the last column of Table 1.

Finally, from the results presented in Table 1 and Figure 3, it is possible to recognize that soil is a heterogeneous porous medium, something that is largely mentioned in the existent literature.

\section{Conclusions}

In the present study, it has been presented the use of free access software for the analysis of pore size distribution of complex porous systems such as soil. It was idealized to be implemented in advanced classes of physics applied to earth, soil, and environmental sciences. The method proposed employs binary digital images, which can be, for instance, uploaded from scientific data repositories. The results presented demonstrated the possibility of the use of inexpensive and practical tools for teaching advanced and modern topics of investigation in applied physics courses.

\section{References}

[1] Z. Yang, X.F. Peng, D.J. Leen and M.Y. Chen, Environ. Sci. Technol. 43, 3248 (2009). 
[2] C.M.P. Vaz, I.C. de Maria, P.O. Lasso and M. Tuller, Soil Sci. Soc. Am. J. 75, 832 (2011).

[3] F.J. Muñoz-Ortega, F. San José Martínez and F.J. Caniego Monreal, Pure Appl. Geophys. 172, 167 (2015).

[4] M.V. Galdos, E. Brown, C.A. Rosolem, L.F. Pires, P.D. Hallett and S.J. Mooney, Sci. Rep. 10, 5072 (2020).

[5] T.R. Ferreira, L.F. Pires, D. Wildenschild, A.M. Brinatti, J.A.R. Borges, A.C. Auler and A.M.H. dos Reis, Geoderma 338, 585 (2019).

[6] R.L. Peyton, C.J. Gantzer, S.H. Anderson, B.A. Haeffner and P. Pfeifer, Water Resour. Res. 30, 691 (1994).

[7] L. Luo and H. Lin, Vadose Zone Journal 8, 233 (2009).

[8] T. Mukunoki, Y. Miyata, K. Mikami and E. Shiota, Soil Earth 7, 929 (2016).

[9] F.A.M. Cássaro, A.N.P. Durand, D. Gimenez and C.M.P. Vaz, Soil Sci. Soc. Am. J. 81, 468 (2017).

[10] H.J. Vogel, Eur. J. Soil Sci. 48, 365 (1997).

[11] L.F. Pires, S.J. Mooney, A.C. Auler, B. Atkinson and C.J. Sturrock, Geoderma 337, 935 (2019).

[12] R. Brewer, Fabric and mineral analysis of soils (John Wiley \& Sons, New York, 1964). 